\title{
Estimation of Critical Parameters in Concrete Production Using Multispectral Vision Technology
}

\author{
Michael E. Hansen ${ }^{1}$, Bjarne K. Ersbøll ${ }^{1}$, Jens M. Carstensen ${ }^{2}$, \\ and Allan A. Nielsen ${ }^{1}$ \\ 1 Informatics and Mathematical Modelling (IMM), \\ Richard Peternsens Plads, Building 321, \\ Technical University of Denmark \\ meh@imm.dtu.dk \\ 2 Videometer A/S, Lyngs $\varnothing$ Allé 3, \\ DK-2970 Hørsholm
}

\begin{abstract}
We analyze multispectral reflectance images of concrete aggregate material, and design computational measures of the important and critical parameters used in concrete production. The features extracted from the images are exploited as explanatory variables in regression models and used to predict aggregate type, water content, and size distribution. We analyze and validate the methods on five representative aggregate types, commonly used in concrete production. Using cross validation, the generated models proves to have a high performance in predicting all of the critical parameters.
\end{abstract}

\section{Introduction}

The importance of concrete in modern society plays an increasing role. Look around you and you will find concrete structures everywhere. Concrete is a composite material which is made up of a filler and a binder [1]. The binder (or cement paste) "glues" the filler together to form a synthetic conglomerate. The constituents used for the binder are cement and water, while the filler can be a fine or coarse aggregate.

$$
(\text { cement }+ \text { water })+\text { aggregate }=\text { concrete }
$$

The key ingredient in concrete production is water. When mixed with cement, water forms a paste that binds the aggregate together. The water causes the hardening of concrete through a process called hydration; a chemical reaction in which the major compounds in cement form chemical bonds with water molecules and become hydrates or hydration products. The role of water is important because the water to cement ratio is the most critical factor in the production of "perfect" concrete. Too much water reduces concrete strength, while too little will make the concrete unworkable. Concrete needs to be workable so that it may 
be consolidated and shaped into different forms (i.e. walls, domes, etc.). Because concrete must be both strong and workable, a careful balance of the cement to water ratio is required when making concrete.

Although a small amount of water is needed to complete the chemical reaction with cement, additional water is necessary to make the concrete workable. As the paste is thinned out with water, its quality is lowered. It will have less strength and less durability. For quality concrete a proper proportion of water and cement is essential. This proportion is called water-cement ratio. The water-cement ratio is determined by dividing the weight in kilograms of the total actual mixing water by the weight in kilograms of cement used in the mix. Especially, when the aggregate is sand or finer particles, an unspecified amount of water is always present. When adding additional water to the concrete, this water has to be taken into account, in order to obtain the optimal water-cement ratio. The "latent" amount of water can vary from $1-10 \%$ of the weight (e.g. approximately 100 liters of water distributed evenly around all grains in $1 \mathrm{~m}^{3}$ of aggregate material). Another critical parameter is the size distribution, or gradation, of the aggregate. This is one of the most influential aggregate characteristics in determining how the concrete will perform. It helps determine almost every important property including stiffness, stability, durability, permeability, workability, fatigue resistance, frictional resistance and resistance to moisture damage.

In order to get the optimal concrete mixture, the critical parameters have to be accounted for online in the concrete production. It is the objective of this study to evaluate the use of vision technology as a tool to estimate the following important critical parameters of aggregate samples: 1) The water content, and 2) the aggregate size distributions.

\section{Materials}

\subsection{Sample Preparation}

The experiment was based on five different types of aggregates (sand/gravel), commonly used when producing concrete. They were chosen to represent as different types as possible: different origin (from lake or hill), different preparation (washed or not), and finally the sand types should be classified for use in different environments.

The next step was to prepare the aggregates to have desired size distributions with desired water contents. First, the samples were prepared to have specific size distributions. It was decided to adapt to the current methods used in the production line. Therefore, three types of size distributions were chosen ("Fine", "Normal" and "Coarse"). One for each aggregate type. To accommodate the fact, that the technician work needed for this task is exhausting, aggregate material having "Fine" and "Coarse" size distributions were only prepared for the the types 1,3 , and 5 , whereas the types 2 and 4 only were represented by the "Normal" size distribution. This choice were based on knowledge about the ma- 


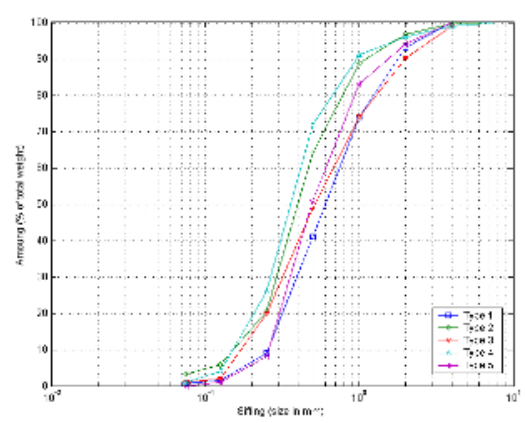

(a)

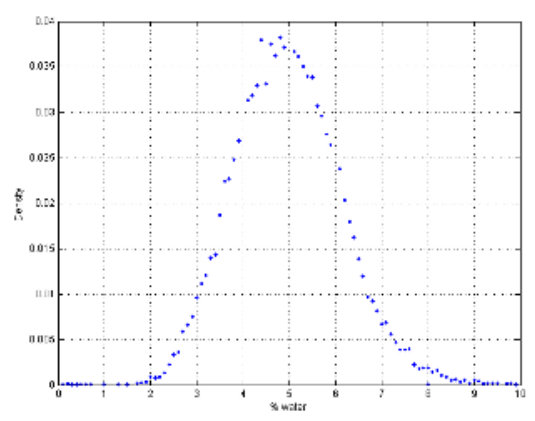

(b)

Fig. 1. (a) Size distribution of the different aggregate types $1, \ldots, 5$. (b) The distribution of water content in aggregates obtained from 23281 samples of different types used in concrete production

terial, hence 1, 3 and 5 should be regarded as representative for 2 and 4 . Figure 1(a) illustrate the size distribution, "Normal", represented for all types.

Finally, for each combination of type and size distribution, samples with a desired water content was prepared. Over the years, the concrete producer, $4 \mathrm{~K}$ Concrete, has manually monitored the amount of water present in the aggregates that they use. In order to have as realistic data material as possible, data about the measured water contents were used to identify the "real world" distribution of water in aggregate material (see Figure 1(b)). Based on this distribution, samples were prepared to have following water contents: $1.25 \%, 2.5 \%, 3.75 \%$, $5.0 \%, 6.25 \%, 7.5 \%$ and $8.75 \%$ of the sample material weight. Establishment of these levels in the samples were based on the following steps: First all samples were dried out in an oven at $105^{\circ} \mathrm{C}$, and finally, from the dried material samples were taken and water were added according the their weight, giving the correct water content.

Triplicates were produced of each combination of type, size distribution and water content, and put into sealed containers. From these containers material was filled into petri dishes and images were acquired (Section 2.2). Since the water content of the triplicates was approximated, the exact content was finally found by drying and weighing, after image acquisition. Finally, we had a total amount of samples of $52 \times 3=156$ (including the replicates).

\subsection{Image Acquisition}

After preparation, the samples were put into petri dishes and digitized using the VideometerLab (illustrated in Figure 2). VideometerLab is a vision-based system for color and texture measurements. The camera is looking through an integrating (Ulbricht) sphere and the petri dishes were placed in an opening on 


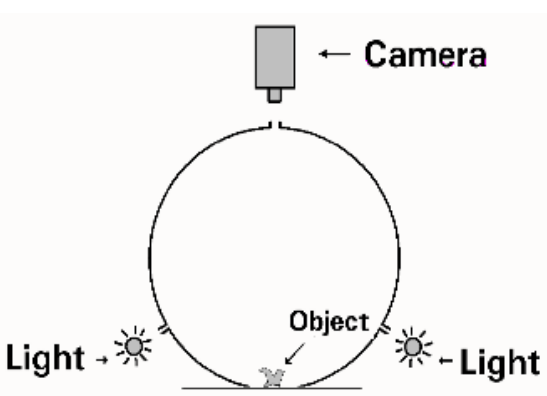

(a) Principle
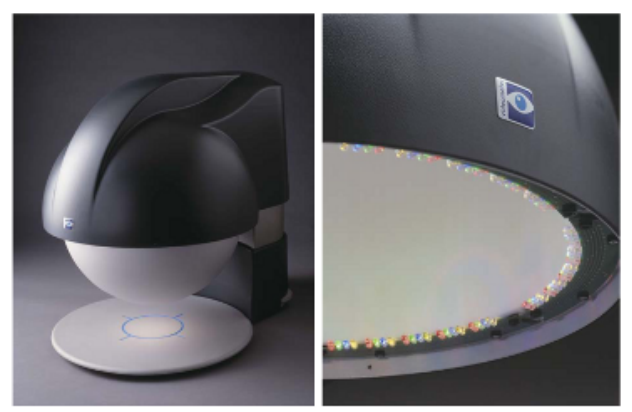

(b) Instrument

Fig. 2. VideometerLab is one implementation of the multispectral vision technology. (a) Illustration of the principle of imaging with an integrating sphere creating uniform illumination. Illumination of the object will come from reflections from the coating on the inner surface of the sphere. (b) The different diodes in the instrument

the opposite side of the sphere (Figure 2(a)). Using this system the dishes receives a uniform and diffuse light, and shading effects, shadows, and gloss-related effects are minimized. Furthermore the geometry of the illumination system is relatively simple to apply in an optical model. This means that the errors that are inherent in the system can be estimated and corrected for.
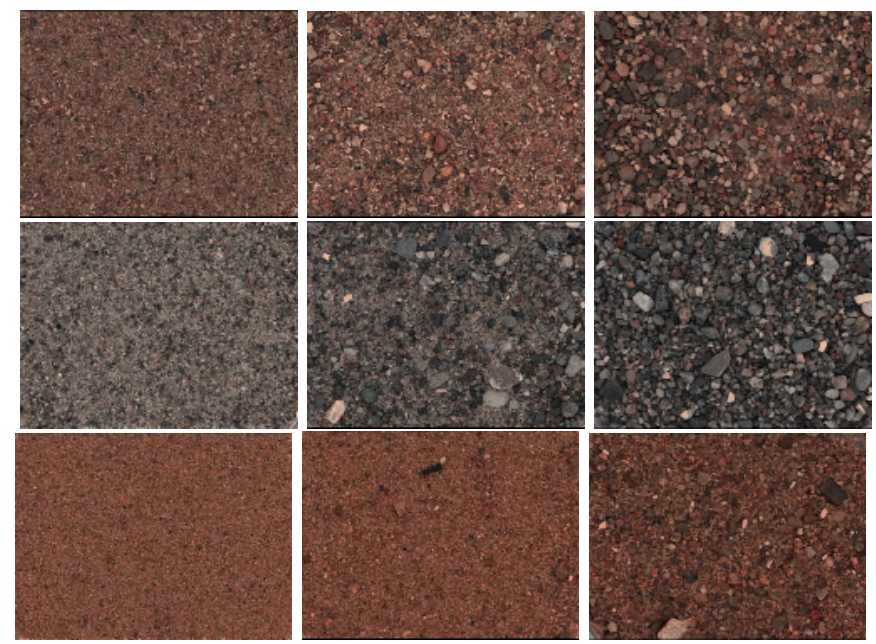

Fig. 3. Examples raw image data after acquisition. Illustration of three types of aggregates with different size distributions. The rows are ordered according to type: 1,3 and 5. The columns are ordered according to size distribution: "Fine", "Normal" and "Coarse". All images have a water content of $2.5 \%$ 
The multispectral measurements are obtained by strobing light emitting diodes (LED) with different spectral characteristics, and the reflectance from the surface of a petri dish is detected by a mega-pixel black-and-white camera producing a high resolution multispectral image.

Using this technology (Figure 2(b)), multispectral reflectance images were captured of all samples, each of which contained 9 frames (1035rows $\times$ 1380 columns $\times 9$ bands $\times 32 \mathrm{bit} /$ pixel): $472 \mathrm{~nm}$ (blue), $515 \mathrm{~nm}$ (green), $592 \mathrm{~nm}(\mathrm{am}-$ ber), $630 \mathrm{~nm}$ (red), $875 \mathrm{~nm}$ (nir[low]), 428nm (ultra blue), $503 \mathrm{~nm}$ (cyan), $612 \mathrm{~nm}$ (orange), 940nm, (nir[high]). In Figure 3 six images captured can be seen. The images illustrate some of the diversity in type and size distribution.

\section{Methods}

During this study, we found that it is necessary to generate models for each of the aggregate types. There were an "overlap" between some of the aggregates in such a way, that two aggregates with different size distributions, could have the approximately same appearance at two different water contents. In order to overcome this, it was decided to have type specific models which led to a significant improvement of the methods. The reflectance bands themselves did not contained all the information, but a combination between them was more describing. The original bands were combined into new ones. The new "bands" were based on the logarithm, difference and the ratio between the original bands. Simple global features were extracted from from all bands: $5 \%, 10 \%, 20 \%, \ldots, 90 \%, 95 \%$ quantiles. Other and more complex measures were evaluated, but it was found that although a slight improvement was obtained, the gain was insignificant compared to processing time and complexity.

\subsection{Identification of Aggregate Type}

Although information about type is present, when the concrete recipes is "chosen" in the factory, we sought for a method to validate and control that the information given is correct. When the features has been extracted and a large number of variables are employed, the risk of obtaining a poor classification increases due to the increased likelihood of noisy variables. A pre-selection methodology was implemented prior to classification, to screen out noisy and non-discriminating features.

Consider the $k=1, \ldots, 5$ types with $n_{1}, \ldots, n_{k}$ multivariate $p$-dimensional observations $\left\{\boldsymbol{X}_{i j}\right\}$, where $i$ is the group index and the $j$ is the observation number. The group means are denoted $\left\{\overline{\boldsymbol{X}}_{1}, \ldots, \overline{\boldsymbol{X}}_{k}\right\}$ and the overall mean is denoted $\overline{\boldsymbol{X}}$, i.e.

$$
\begin{aligned}
\overline{\boldsymbol{X}}_{\boldsymbol{i}} & =\frac{1}{n_{i}} \sum_{j=1}^{n_{i}} \bar{X}_{i j}, \quad i=1, \ldots, k, \\
\overline{\boldsymbol{X}} & =\frac{1}{N} \sum_{i=1}^{k} \sum_{j=1}^{n_{i}} \bar{X}_{i j}, \quad i=1, \ldots, k \quad \text { where } \quad N=\sum_{i=1}^{k} n_{i} .
\end{aligned}
$$


As in a one way analysis of variance (ANOVA) the total sum of squares matrix, $\boldsymbol{\Sigma}_{\boldsymbol{T}}$, the between groups matrix, $\boldsymbol{\Sigma}_{\boldsymbol{B}}$, and the within groups, $\boldsymbol{\Sigma}_{\boldsymbol{W}}$, are defined by

$$
\begin{aligned}
& \boldsymbol{\Sigma}_{B}=\sum_{i=1}^{k} n_{i}\left(\overline{\boldsymbol{X}}_{i}-\overline{\boldsymbol{X}}\right)\left(\overline{\boldsymbol{X}}_{i}-\overline{\boldsymbol{X}}\right)^{T} \\
& \boldsymbol{\Sigma}_{W}=\sum_{i=1}^{k} \sum_{j=1}^{n_{i}}\left(\overline{\boldsymbol{X}}_{i j}-\overline{\boldsymbol{X}}_{i}\right)\left(\overline{\boldsymbol{X}}_{i j}-\overline{\boldsymbol{X}}_{i}\right)^{T}, \\
& \boldsymbol{\Sigma}_{T}=\boldsymbol{\Sigma}_{B}+\boldsymbol{\Sigma}_{W}=\frac{1}{N} \sum_{i=1}^{k} \sum_{j=1}^{n_{i}}\left(\overline{\boldsymbol{X}}_{i j}-\overline{\boldsymbol{X}}\right)\left(\overline{\boldsymbol{X}}_{i j}-\overline{\boldsymbol{X}}\right)^{T} .
\end{aligned}
$$

Wilks' lambda can be defined as the ratio of the determinant of the betweengroup variance, $\boldsymbol{\Sigma}_{W}$, to the determinant of the total variance, $\boldsymbol{\Sigma}_{T}$ for each feature to obtain an initial set of discriminating features [5]

$$
\Lambda=\frac{\left|\boldsymbol{\Sigma}_{W}\right|}{\left|\boldsymbol{\Sigma}_{T}\right|} .
$$

Wilks' lambda, $\Lambda$, can be transformed into an $F$-distribution,

$$
V_{j}=-\left[(n-1)-\frac{p+2}{2}\right] \ln (\Lambda),
$$

in which $n$ is the number of samples, and $p$ the number of predictor variables (in $\left.\boldsymbol{X}_{i j}\right)$. This allows the selection of discriminatory features with an appropriate confidence level $[4,6]$. The test is analogous to to the $F$-test used to test the significance of a regression model. The statistic $V_{j}$ approximately follows a $\chi^{2}$ distribution with $p$ degrees of freedom. In order to further reduce the amount of features and also remove redundant information, principal component analysis (PCA) [7] was applied and the principal components (PC's) explaining 95-99\% of the variation was used in the further analysis.

After the elimination/reduction of features, that were found to be insignificant on a $5 \%$ significance level, canonical discriminant analysis (CDA) [7] of the remaining features were used to obtain the proper projection of data based on "optimal separation". We are looking for projections that maximize the ratio of the variation between groups and the variation within groups. The idea of maximizing this ratio was proposed by Fisher in 1936 [3], and this ratio equals the Rayleigh coefficient (or Fisher ratio)

$$
J(\boldsymbol{w})=\frac{\boldsymbol{w}^{T} \boldsymbol{\Sigma}_{B} \boldsymbol{w}}{\boldsymbol{w}^{T} \boldsymbol{\Sigma}_{W} \boldsymbol{w}}
$$

i.e. the transformation is defined by the eigenvectors $\boldsymbol{w}$ of $\boldsymbol{\Sigma}_{\boldsymbol{B}}$ with respect to $\boldsymbol{\Sigma}_{\boldsymbol{W}}$ and is found by $\boldsymbol{\Sigma}_{B} \boldsymbol{w}=\lambda \boldsymbol{\Sigma}_{W} \boldsymbol{w}$. The new variates are found by $\boldsymbol{Y}=$ $\boldsymbol{w}^{T}\left(\boldsymbol{X}_{i j}-\overline{\boldsymbol{X}}\right)$. 


\subsection{Estimation of Water Content}

The explaining variates with respect to water content may differ from those for type. Therefore a new feature elimination procedure was applied. The dependent variables are now on a continuous scale, and a slightly different approach than the one in Section 3.1 has to be followed. Based on regression analysis [7] only features were selected, that could be proven to contain information. Redundant information was removed by applying PCA and the PC's retaining $95 \%$ of the variance was kept for further analysis. The PC's were then used as input to a final regression analysis, modelling the water content of the samples, purely based on image information.

\subsection{Estimation of Aggregate Size Distribution}

Let the values in a multispectral be described by $I_{n}(x, y), n=1, \ldots, N$, where $n=1, \ldots, 9$ is a specific band, and $I(x, y)$ the corresponding pixel (reflectance) at the coordinate $(x, y)$. Since only the structural information is of interest, band information is reduced through an average

$$
\tilde{I}(x, y)=\sum_{n=1}^{N} \omega_{n} I_{n}(x, y)
$$

where $\omega_{n}$ is a weight put on each of the bands. These weights can be found by by PCA or a similar method, but in this study all weights were $\omega_{n}=\frac{1}{N}$. Finally, we center and standardize $\tilde{I}(x, y)$ to have $\mathrm{E}[\tilde{I}(x, y)]=0$ and $\mathrm{V}[\tilde{I}(x, y)]=1$.

Next, we apply a scale-space approach and convolve with a Gaussian kernel where $\sigma$ is specifying the scale. The result can be seen as low-pass filtering that removes finer details as the scale, $\sigma$, increases. The filtered image is obtained by convolution

$$
h(r ; \sigma)=\frac{1}{\sqrt{2 \pi \sigma^{2}}} e^{-\frac{r^{2}}{2 \sigma^{2}}}, \quad \tilde{I}_{\sigma}(x, y)=\left(\tilde{I}_{\sigma}(x, y) * h_{\hat{x}}\right) * h_{\hat{y}}
$$

where $h_{\hat{x}}$ and $h_{\hat{y}}$ are the horizontal and vertical 1D separable kernels of the Gaussian filter. Features were extracted from $\tilde{I}_{\sigma}(x, y)$. The features were simple statistics: mean, standard deviation, skewness and kurtosis. In order to evaluate if the statistics were due to edges from many smaller objects or from one large or several larger objects in $\tilde{I}_{\sigma}(x, y)$, the mean and standard deviation of the gradient

$$
G(x, y)=\left|\nabla \tilde{I}_{\sigma}(x, y)\right|=\left|\left(\frac{\delta}{\delta x} \hat{x}+\frac{\delta}{\delta y} \hat{y}\right) \tilde{I}_{\sigma}(x, y)\right|
$$

did show to give significant improvement to the method.

The strategy we have chosen to follow from here, is to predict the amount of aggregate material remaining in each of the sieves (for each types). Again, we end up with many features, and we apply the same strategy as when predicting the water contents. Having removed the insignificant variates, the PCA was used to reduce the number of features and remove redundancy. And the PC's retaining $99.9 \%$ of the variance were used in the final regression analysis. 


\section{Results}

First step was to find a model for aggregate type. After the type was identified, aggregate type specific models were applied in order to predict the water content, and size distribution. The following results are based on a full-cross validation (leave-one out), which means that we took one (or more) samples out before training the models. Finally the sample(s) were identified, and the critical parameters were estimated.

Figure 4 show the cross-validation results of the type classification. As can be seen, all types are identified with no errors.

After identifying the aggregate type, the water content was predicted. Figure 5 shows the relations between the measured water content (Figure 5(a)) and the

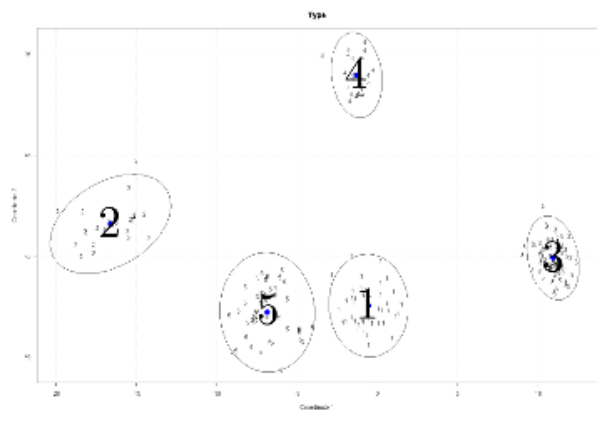

Fig. 4. The first and second canonical discriminant function of the aggregate types. The results are obtained by a leave one out cross-validation. The figure shows, that there is a clear and consistent separation between the five types

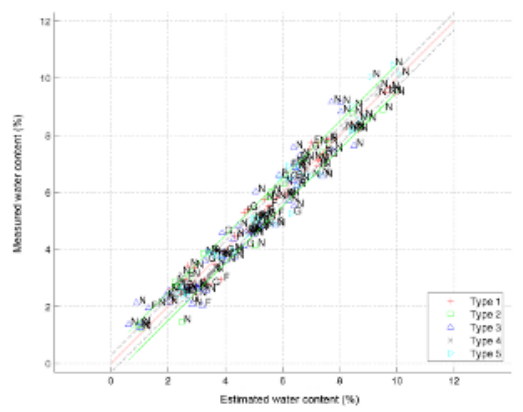

(a) Measured vs. predicted values.

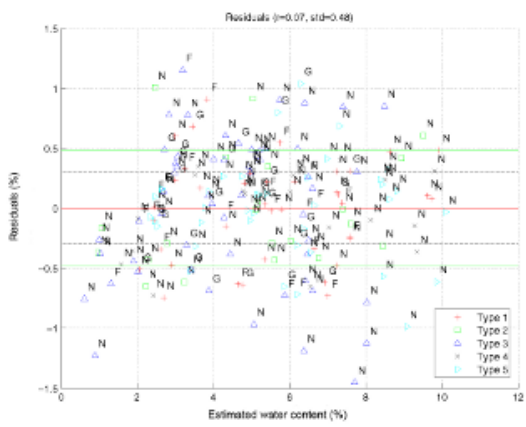

(b) Residuals vs. predicted values.

Fig. 5. Results after estimation of water content. The results are obtained by leave one out cross-validation 

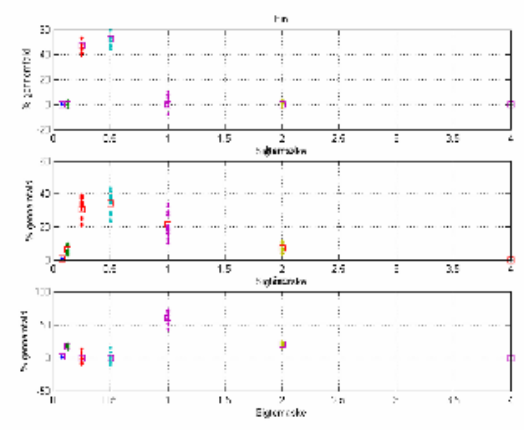

(a)

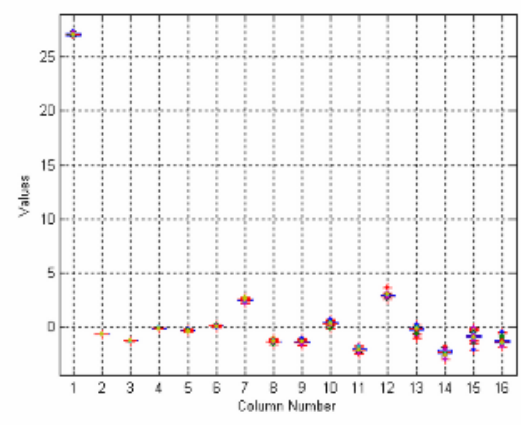

(b)

Fig. 6. Results after size distribution estimation for aggregate type 1. (a) Showing the size distribution values (predicted: $\bullet$, laboratory value: $\square$ ) of sieved aggregate material (relative to the total amount in all sieves). (b) The regression weights. All results are obtained by leave one out cross-validation

residuals (Figure 5(a)) as function of the predicted values. From the figures it can be seen, that there is a clear relation between the estimated and the predicted water contents. The standard deviation of the residuals is $\sigma=0.48 \%$.

Finally, the size distributions were estimated. Figure 6 show the result for the one of the types (type 1 ). Figure $6(\mathrm{a})$ shows the predicted values $(\bullet)$ together with the in laboratory measured value $(\square)$. From the figures we conclude, that there is a large agreement between the predicted and measured values. Figure 6 (b) plots the model weights applied on to the 15 PC's that are input to the regression model. Whereas some of the PC's could be regarded as insignificant for some of the aggregate types, they were proven to be significant for other types.

The standard deviations for the residuals for all sieves and all types were lying in the range of $\sigma \in\{0.04 \% ; 7.24 \%\}$ when evaluating the triplicates as separate observations. When pooling the triplicates into one observation the standard deviations fell to be in the range of $\sigma \in\{0.03 \% ; 5.2 \%\}$

\section{Discussion}

In this study, we have shown that it is possible to estimate the critical parameters related to aggregates in concrete production using multispectral vision technology. Reflectance images are captured, and simple low-level features calculated and used as parameters in models describing both water content and size distribution. Although prior knowledge about aggregate type was proven to be necessary in order to obtain satisfying results, the types could be perfectly identified. Based on type specific models, estimates of water content and size distribution were obtained showing high proficiency. 
Although the performance of the methods was high, many question still has to be answered, i.e. what are the significant features and how can they be interpreted? Also, the bands when estimating the size distributions are pooled. It might be, that there are more information hidden within each of the single bands.

The methods suggested are well suited to implemented in an industrial application. The features are simple, robust and easy to be calculate. Further studies have been planned, in order to reveal further knowledge about these and other questions.

\section{Acknowledgments}

This work was supported by The Danish Ministry of Science, Technology and Innovation. We would like to thank Dorthe Mathiesen (Danish Technological Institute) and Freddie Scheye $(4 \mathrm{~K})$ for constructive discussions and Finn Østergaard for skillful technical assistance.

\section{References}

1. S. Mindess and J.F. Young, Concrete. Prentice-Hall, Inc., Englewood Cliffs, NJ, 1981.

2. M. R. Rixom and N. P. Mailuaganam, Chemical Admixtures for Concrete. R. \& F.N. Spon, NY, 1986.

3. R.A. Fisher (1936), "The use of multiple measurements in taxonomic problems", Annals of Eugenics 7, 179-188.

4. M. S. Bartlett, "The Statistical Significance of Canonical Correlations", Biometrika, Vol. 32, No. 1. (Jan., 1941), pp. 29-37

5. S. S. Wilks, "Certain Generalizations in the Analysis of Variance", Biometrika, Vol. 24, No. 3/4. (Nov., 1932), pp. 471-494.

6. D. N. Lawley, "Tests of Significance in Canonical Analysis", Biometrika, Vol. 46, No. 1/2. (Jun., 1959), pp. 59-66.

7. J. Lattin, J. D. Carroll and P. E. Green, "Analyzing Multivariate Data", Brooks/Cole, 2003. 